\title{
PATHOLOGICAL MODULES OVER TAME RINGS
}

\section{SHEILA BRENNER AND CLAUS MICHAEL RINGEL}

\section{Introduction}

What are pathological modules?

(i) an indecomposable module whose minimal generating sets are large (say of cardinal greater than or equal to $c$, where $c$ is any fixed cardinal less than $\aleph_{\imath}$, the first strongly inaccessible cardinal)

(ii) a module whose minimal generating sets are arbitrarily large and which cannot be written as an infinite direct sum of non-zero submodules

(iii) a module without non-zero indecomposable direct summands

(iv) a module which, for every integer $q \geqslant 2$, can be written as the direct sum of $q$ indecomposable submodules, but not as the direct sum of infinitely many submodules

(v) indecomposable modules $M_{0}, M_{1}, M_{2}$ with

$$
M_{0} \oplus M_{1} \simeq M_{0} \oplus M_{2} \text { but } M_{1} \neq M_{2}
$$

(vi) a module $M$ such that

$$
\oplus_{n} M \simeq \oplus_{m} M \quad \text { if and only if } n \equiv m(\bmod q),
$$

where $q \geqslant 2$ is a fixed integer.

All such pathological modules exist for the free associative algebra $k\langle x, y\rangle$ in two (non-commuting) variables over a field $k$. They therefore occur also for any $k$-algebra $A$ for which there exists a full embedding of the module category ${ }_{k\langle x, y\rangle} \mathfrak{M l}$ into the module category ${ }_{A} \mathfrak{M}$. Indeed, in order to ensure the existence of pathological $A$-modules we need only require that a full subcategory of ${ }_{A} \mathfrak{M}$ is representation equivalent to $k\langle x, y\rangle \mathfrak{M}$, i.e. that $A$ is "wild", or of wild representation type. At the other extreme, if $A$ is a finite-dimensional $k$-algebra which has only finitely many indecomposable modules, then every indecomposable module is finite-dimensional and every module is a direct sum of indecomposable ones. Thus, for such $A$, there are no pathological modules at all.

In this paper we consider the remaining case of an algebra of tame representation type-that is, the case of an algebra $A$ which has no full subcategory representation equivalent to $k\langle x, y\rangle, \mathfrak{M}$, but which has infinitely many indecomposable representations. An example of a tame algebra is $k[t]$, the polynomial ring in one variable over the field $k$. For all known finite-dimensional tame algebras there is a full subcategory of $A$-modules which is representation equivalent to ${ }_{k[t]} \mathfrak{P}$. Now the question is: what kinds of pathological modules does a tame algebra $A$ have? We shall show that, even for $A=k[t]$, the answer is: all types of pathological modules. From this result for $k[t]$-modules it follows that all known finite-dimensional tame algebras also have all types of pathological modules.

Received 24 July, 1975.

[J. London Math. Soc. (2), 14 (1976), 207-215] 
We follow closely Corner's answer [3] to the corresponding question for abelian groups, that is $Z$-modules. Thus we consider the case of a principal ideal domain $R$, generalising Corner's result $(R=Z)$, and take particular interest in the case $R=k[t]$. From now on $R$ will denote a (commutative) principal ideal ring. We assume further that $R$ is neither a field nor a complete valuation ring, since the results are vacuous in these cases.

Corner shows that any ring with suitable additive structure can be realised as the endomorphism ring of an abelian group. We generalise this to give a realisation of a prescribed $R$-algebra $A$ with suitable $R$-module structure as the endomorphism ring of an $R$-module. The construction may be regarded as giving a "realisation" in the category of $R$-modules of the full subcategory of ${ }_{A} \mathfrak{M}$ with one object ${ }_{A} A$. It can be generalised easily to give a "realisation" of a larger category $\mathscr{C}$ as a category of $R$-modules.

What kind of categories $\mathscr{C}$ can be realised? We start with a nearly arbitrary $R$-algebra $A$, the main restriction being that $A$ can be centrally generated (see $\$ 2$ ) over $R$ by less than $\aleph_{1}$ elements. Then $\mathscr{C}$ is the full subcategory of all $A$-modules which, when regarded as $R$-modules, belong to a suitable class of $R$-modules-one which is "Szekeres incomplete". (This imposes a further, and last, condition on $A$.)

Definition. A class $\mathfrak{R}$ of $R$-modules is called Szekeres incomplete if each $R$-module in $\mathfrak{N}$ is torsion-free, and there exists a set $\mathscr{P}$ of prime elements of $R$ such that

(i) each $R$-module $M$ in $\mathfrak{N}$ is $\mathscr{P}$-Hausdorff (that means $\bigcap_{p \in \mathscr{P}, n \in \mathbb{N}} p^{n} M=0$ ),

(ii) for each $p \in \mathscr{P}, \hat{Q}_{p}$ has transcendence degree at least 1 over $S_{p}(\mathfrak{N})$, where $\hat{Q}_{p}$ is the completion of the quotient field $Q$ of $R$ with respect to the $p$-adic topology, and $S_{p}(\mathfrak{N})$ is the $p$ th Szekeres field of $\mathfrak{N}$. (As will be seen from the proof of Theorem 1, we require, in fact, only the existence of three elements of $\hat{Q}_{p}$ which are quadratically independent over $S_{p}(\mathfrak{N})$.)

The Szekeres field $S_{p}(\mathfrak{N})$ of $\mathfrak{N}$ measures how many elements of $\hat{Q}_{p}$ are necessary to generate all modules $M$ of $\mathfrak{N}$ when using $Q_{p}$-independent elements of $M$. For the precise definition the reader is referred to $\$ 2$. We note here two examples: (i) If $\mathfrak{N}=\mathscr{F}$ is the class of all free $R$-modules, then $S_{p}(\mathfrak{N})=Q$ for all primes $p$. Thus $\mathscr{F}$ is Szekeres incomplete provided that there exists a prime $p$ for which $Q \subseteq \widehat{Q}_{p}$ has transcendence degree at least 1 . (ii) If $\mathfrak{N}$ contains only countably many $R$-modules and each of them is countably generated, then $Q \subseteq S_{p}(\mathfrak{N})$ is a countable field extension. Hence for $R=k[t]$, both the class $\mathscr{F}$ of all free $R$-modules and any countable set $\mathfrak{R}$ of countably generated torsion-free, Hausdorff $R$-modules are Szekeres incomplete classes.

THEOREM 1. Let $\mathbb{R}$ be a principal ideal domain, $\mathfrak{N}$ a Szekeres incomplete class of $R$-modules, and let $A$ be an $R$-algebra centrally generated over $R$ by $c<N$, elements. Denote by $\mathscr{C}=\mathscr{C}(\mathfrak{N}, A)$ the full subcategory of all $A$-modules ${ }_{A} X$ such that the corresponding $R$-module ${ }_{R} X$ belongs to $\mathfrak{N}$. Then there exists a full exact embedding $T$ of $\mathscr{C}$ into ${ }_{R} \mathfrak{M}$ such that, if $M \in \mathscr{C}$ has $R$-rank $m$, then $T(M)$ has rank $\mathrm{cm}$.

Here, exact embedding means that sequences of $A$-modules in $\mathscr{C}$, which are exact in ${ }_{A} \mathfrak{M}$, are mapped to exact sequences in ${ }_{R} \mathfrak{M}$. To repeat what we said earlier, this theorem says that we can realise certain categories $\mathscr{C}$ as full subcategories of ${ }_{R} \mathfrak{M}$.

Note that the requirement that $\mathfrak{R}$ is Szekeres incomplete imposes a condition on the $R$-algebras $A$ for which $\mathscr{C}(\mathfrak{N}, A)$ is non-trivial. 
Special cases of Theorem 1 have been obtained by other authors. They include

(i) (Corner [3]). $A$ is torsion-free, reduced and countably generated as an $R$-module and $\mathfrak{N}$ is a class of countably generated $R$-modules.

(ii) (Warfield [12]). $R$ is a discrete valuation ring whose completion $R$ has uncountable transcendence degree over $R, A$ is countably generated, torsionfree and reduced as an $R$-module and $\mathfrak{N}$ is a class of countably generated $R$-modules.

Theorem 1 will be deduced from the special case $A=R\langle x, y\rangle$, the free $R$-algebra on two generators $x$ and $y$, by using the following result:

THEOREM 2. Let $B$ be a commutative ring and $A$ a B-algebra centrally generated over $B$ by $c<\aleph_{1}$, elements. Then there exists a full exact embedding $F$ of the category

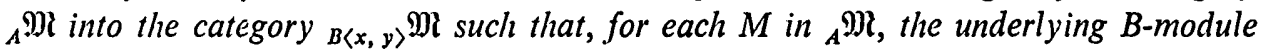
structure of $F\left({ }_{A} M\right)$ is the direct sum of $c+2$ copies of ${ }_{B} M$.

This theorem is an easy corollary of Corner's five submodule theorem [6]. A proof of it, applicable to the case when $B$ is not necessarily commutative, will be sketched in $\S 4$.

\section{Preliminaries}

(a) Topological ideas.

Let $R$ be a principal ideal domain and $p$ be a prime in $R$. We denote by $R_{p}$ the ring of fractions with denominators prime to $p$ and by $\widehat{R}_{p}$ its completion in the $p$-adic topology. We write $\hat{Q}_{p}$ for the ring of fractions of $\widehat{R}_{p}$, and $Q$ for the ring of fractions of $R$.

The natural topology on $R$ is the topology in which $\{q R: 0 \neq q \in R\}$ is a basis of neighbourhoods of zero. We write $\widehat{R}$ for the completion of $R$ in this topology. It is possible to represent $\widehat{R}$ in the form $\widehat{R}=\prod_{p} \widehat{R}_{p}$, where the product is taken over all
primes $p$ in $R$.

If $M$ is a torsion-free $R$-module, Hausdorff in (the topology induced by) the natural topology on $R$ (so that $M$ has no divisible submodules, i.e. $M$ is reduced), then the completion of $M$ in this topology is denoted $\hat{M}$ and is naturally an $\widehat{R}$-module.

For any $R$-module $M$, let $\mathscr{M}_{p}$ denote the submodule of elements of infinite $p$-height in $R_{p} \otimes M$. Then $M_{p}=R_{p} \otimes M / \mathscr{M}_{p}$ is reduced, and we denote by $\pi_{p}$ the natural projection. We write $\hat{M}_{p}$ for the $p$-adic completion of $M_{p}$.

If $M$ is Hausdorff in the natural topology, then $\hat{M}$ can be represented in the form $\hat{M}=\prod_{p} \hat{M}_{p}$ and the map $M \rightarrow \hat{M}, m \mapsto\left(\pi_{p} m\right)$ is an injection.

(b) Purity.

Let $M$ be a torsion-free Hausdorff $R$-module. The sub-module $N$ of $M$ is said to be pure in $M$ if $q M \cap N=q N$ for all $q \in R$.

If $N$ is pure in $M$ then the natural topology on $N$ coincides with the topology induced by the natural topology on $M$, i.e. $N$ is a topological submodule.

For any $M, M$ is pure in $\hat{M}$ and, if $N$ is a pure submodule of $\hat{M}$ and $M \subseteq N$, then $\hat{N}=\hat{M}$.

The corresponding concept for $R_{p}$-modules is called $p$-purity. 
(c) The Szekeres field.

Let $\mathfrak{N}$ be a category of torsion-free $R$ modules and $p$ be a prime in $R$. For each $M \in \mathfrak{N}$ let $\left\{x_{i}^{M}\right\}_{i \in I^{M}}$ (where $I^{M}$ is an index set) be a set of generators for $M_{p}$ as an $R_{p}$-module. Choose $J^{M} \subseteq I^{M}$ so that $\left\{x_{j}^{M}\right\}_{j \in J^{M}}$ is maximal with respect to independence (in $\hat{M}_{p}$ ) over $\widehat{R}_{p}$. Then in $Q \otimes \widehat{M}_{p}$ we have, for all $i \in I^{M}$,

$$
x_{i}^{M}=\sum_{j \in J^{M}} \pi_{i j} x_{j}^{M}, \text { where } \pi_{i j} \in \hat{Q}_{p} \text { and almost all } \pi_{i j}=0 .
$$

Let $S_{p}(\mathfrak{N})$ be the field generated over $Q$ by $R_{p}$ and $\left\{\pi_{i j}: i \in I^{M}, j \in J^{M}, M \in \mathfrak{N}\right\}$. Then $S_{p}(\mathfrak{N})$ depends only on $\mathfrak{N}$ and is called the Szekeres field [10] of $\mathfrak{N}$ for the prime $p$.

\section{(d) Centralising generators.}

Definition. Let $B$ be a ring. The ring $A$ is said to be (left) centrally generated over $B$ by a subset $\Gamma \subseteq A$ if

(i) there is a ring homomorphism $B \rightarrow A$, so that $A$ can be regarded as a left (or right) $B$-module, and

(ii) $\left\{\theta \in \operatorname{End}_{B} A: \theta \gamma=\gamma \theta\right.$ for all $\left.\gamma \in \Gamma\right\}=A_{R}$, the ring of right translations of $A$ by elements of itself (so that $A_{R}$ is isomorphic to $A$ ). The subset $\Gamma$ is called a set of (left) centralising generators.

Notice that, even when $B$ is commutative, a set $\Gamma$ of centralising generators of $A$ is not necessarily a set of generators in the usual sense (though the converse is true). For example, if $k$ is a field, the field $k(t)$ of rational functions of $t$ is centrally generated over $k$ by $\{t\}$. This concept turns out to be exactly what is required in the following sections.

\section{Proof of Theorem 1}

It follows from Theorem 2 that it is sufficient to consider the case $c=2$. (The last part of the statement of Theorem 1 in the case when $m$ and $c$ are both finite is obtained by an easy modification of the proof.) The argument follows very closely Orsatti's proof of Corner's theorem [8].

Throughout this section we use the notation introduced in $\$ 2$.

We prove first the local case of Theorem 1 (for the case $c=2$ ) in the form

Proposition 1. Let $\mathfrak{M}_{p}$ be a Szekeres incomplete class of $R_{p}$-modules and $A_{p}$ be an $R_{p}$-algebra centrally generated over $R_{p}$ by $\left\{\mu_{p}, v_{p}\right\}$. Let $\mathscr{C}_{p}$ be the full subcategory of $A_{p}$-modules which, as $R$-modules, belong to $\Re_{p}$. Then there exists a full exact embedding $T_{p}$ of $\mathscr{C}_{p}$ into ${ }_{R_{p}} \mathfrak{M}$ such that, if $M \in \mathscr{C}_{p}$ has $R_{p}$-rank $m$, then $T_{p}(M)$ has rank $2 m$.

Proof. Since $\mathfrak{N}_{p}$ is Szekeres incomplete, $\widehat{Q}_{p}$ has transcendence degree at least 1 over $S_{p}=S_{p}\left(\mathfrak{N}_{p}\right)$. Hence, there exist elements $a_{i}^{(p)} \in R_{p},(i=1,2,3)$, which are quadratically independent over $S_{p}$, i.e. if

$$
\lambda+\sum_{i=1}^{3} \lambda_{i} a_{i}^{(p)}+\sum_{1 \leqslant j \leqslant i}^{3} \lambda_{i j} a_{i}^{(p)} a_{j}^{(p)}=0
$$

with $\lambda, \lambda_{i}, \lambda_{i j}(1 \leqslant j \leqslant i \leqslant 3)$ elements of $S_{p}$, then

$$
\lambda=\lambda_{i}=\lambda_{i j}=0 \quad(1 \leqslant j \leqslant i \leqslant 3) .
$$


Write $\alpha_{1}^{(p)}=1_{A_{n}}, \alpha_{2}{ }^{(p)}=\mu_{p}, \alpha_{3}{ }^{(p)}=v_{p}$ and, for each $M \in \mathscr{C}_{p}$, define

$$
M^{\prime}=\left\{\sum_{i=1}^{3} a_{i}^{(p)} \alpha_{i}^{(p)} m: m \in M\right\} .
$$

Then $M^{\prime}$ is an $R_{p}$-submodule of the $p$-adic completion $\hat{M}$ of $M$. Let $T_{p}(M)$ be the $p$-pure submodule of $\hat{M}$ generated by $M$ and $M^{\prime}$.

It is easily verified that $T_{p}: \mathscr{C}_{p} \rightarrow{ }_{R_{p}} \mathfrak{M}$ has the required properties.

We now return to the global case. Since $A$ and $M \in \mathscr{C}$ are $R$-modules, we may define $\hat{M}, A_{p}, M_{p}, \hat{M}_{p}$ and $\pi_{p}$ as in $\S 2$ for any prime $p \in R$. If $R_{p} \otimes A$ is divisible, i.e. if $p \notin \mathscr{P}$, then $A_{p}=0$ and $M_{p}=0$. Thus, we may write $\hat{M}=\prod_{p \in \mathscr{P}} M_{p}$.

For each $p \in \mathscr{P}$ we write $\alpha_{1}{ }^{(p)}=1_{A_{p}}, \alpha_{2}{ }^{(p)}=\pi_{p} \mu, \alpha_{3}{ }^{(p)}=\pi_{p} v$, where $\mu$ and $v$ are centralising generators of $A$ over $R$, and choose $a_{i}^{(p)} \in \widehat{R}_{p},(i=1,2,3)$, quadratically independent over $S_{p}$.

For $M \in \mathscr{C}$, define

$$
M^{\prime}=\left\{\left(\sum_{i=1}^{3} a_{i}^{(p)} \alpha_{i}^{(p)} \pi_{p} m\right)_{p \in \mathscr{P}}: m \in M\right\} .
$$

Then $M^{\prime}$ is an $R$-submodule of $\hat{M}$. Let $T(M)$ be the pure submodule of $\hat{M}$ generated by $M$ and $M^{\prime}$. Then it is easily verified that $T: \mathscr{C} \rightarrow{ }_{R} \mathfrak{M}$ is a full exact embedding.

\section{Proof of Theorem 2}

Let $B$ be a ring. Denote by $\Lambda_{6}(B)$ the ring generated over $B$ by 11 elements $e_{00}, e_{0 i}, e_{i i}(1 \leqslant i \leqslant 5)$ which commute with elements of $B$ and satisfy the matrix identities $e_{i j} e_{k l}=\delta_{j k} e_{i l}$. Then the category ${ }_{\Lambda_{6}(B)} \mathfrak{M}$ of $\Lambda_{6}(B)$-modules is naturally equivalent to the category of $B$-module diagrams over the diagram-scheme:

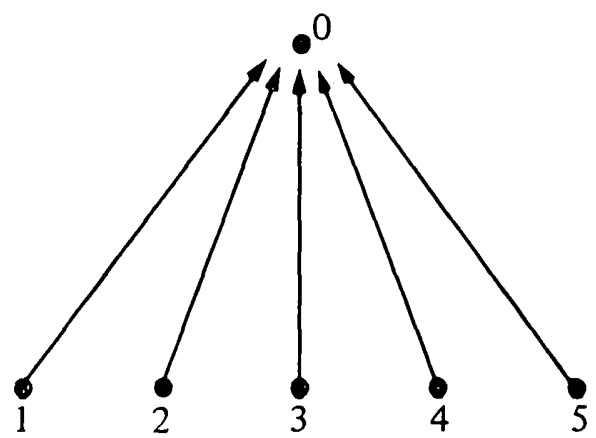

The category $\Delta$ of $B$-modules with five distinguished submodules is the subcategory of diagrams in which all the diagram morphisms are injective. We may thus regard $\Delta$ as embedded (fully and exactly) in ${ }_{\Lambda_{6}(B)} \mathfrak{M}$.

The proof of Theorem 2 consists in showing the existence of full, exact embeddings of ${ }_{A} \mathfrak{M}$ into ${ }_{\Lambda_{6}(B)} \mathfrak{M}$ (Proposition 2) and of ${ }_{\Lambda_{6}(B)} \mathfrak{M}$ into ${ }_{B\langle x, y\rangle} \mathfrak{M}$, which follows from Lemma 1.

LEMMA 1. Let $A$ be a ring centrally generated over $B$ by a finite set of $n$ elements. Then there is full exact embedding $F$ of the category ${ }_{A} \mathfrak{M}$ of $A$-modules into the category of $B\langle x, y\rangle$-modules such that, if $M \in_{A} \mathfrak{M}$ is generated over $B$ by melements, then $F(M)$ is generated over $B$ by $(n+2) m$ elements. (If $B$ is an integral domain and the rank of ${ }_{B} M$ is $m^{\prime}$, the rank of ${ }_{B} F(M)$ is $\left.(n+2) m^{\prime}\right)$. 
This is proved in [2] for the case when $B$ is a field. As in that case the proof is straightforward once one has constructed $F(M)$ as the direct sum of $(n+2)$ copies of $M$ with $x$ and $y$ acting, respectively, as

$$
\left(\begin{array}{lllllll}
0 & 1 & 0 & . & . & . & 0 \\
0 & 0 & 1 & . & . & . & 0 \\
. & . & . & & & & . \\
. & . & . & & & & . \\
. & . & . & & & & . \\
0 & 0 & 0 & . & . & . & 1 \\
0 & 0 & 0 & . & . & . & 0
\end{array}\right) \text { and }\left(\begin{array}{ccccccccc}
0 & 0 & 0 & . & . & . & 0 & 0 & 0 \\
1 & 0 & 0 & . & . & . & 0 & 0 & 0 \\
\mu_{1} & 1 & 0 & . & . & . & 0 & 0 & 0 \\
0 & \mu_{2} & 1 & . & . & . & 0 & 0 & 0 \\
. & . & . & & & & . & . & . \\
. & . & . & & & & . & . & . \\
. & . & . & & & & . & . & . \\
0 & 0 & 0 & . & . & . & \mu_{n} & 1 & 0
\end{array}\right)
$$

where $\mu_{1}, \ldots, \mu_{n}$ are the endomorphisms of $M$ induced by the centralising generators of $A$, and $1=1_{M}$.

This establishes Theorem 2 for the case when $c$ is finite. The case of $c$ infinite follows immediately from this and

Proposition 2. Let $B$ be a ring, $c<\aleph_{1}$, an infinite cardinal and $A$ a ring centrally generated over $B$ by a set $\Gamma$ of generators with $|\Gamma| \leqslant c$. Then there exists a free abelian group $V$ of rank $c$ and a full exact embedding $L:{ }_{A} \mathfrak{N} \rightarrow{ }_{\Lambda_{6}(B)} \mathfrak{M N}$ such that, if $M \in{ }_{A} \mathfrak{M}$, then

$$
e_{00} L(M)={ }_{B} M \otimes V .
$$

This is essentially Theorem 2.3 of Corner's paper [6]. The proof sketched in the remainder of this section derives entirely from that paper. We quote special cases of results from it, sometimes with minor modifications, and sketch proofs only when a construction used is helpful in clarifying our argument.

Lemma 2. Let $B, c, A, \Gamma$ be as in Proposition 2, and $T$ be a free abelian group of rank c. Then there exists a free direct summand $T^{\prime}$ of $T$ and a functor $P:{ }_{A} \mathfrak{M} \rightarrow{ }_{B} \mathfrak{M}$ which assigns to $M \in{ }_{A} \mathfrak{M}$ a B-submodule $P(M) \subseteq M \otimes T$ such that

and, for $M, N \in \in_{A} \mathfrak{M}$

$$
P(M) \oplus\left(M \otimes T^{\prime}\right)=M \otimes T \text { in }{ }_{B} \mathfrak{M}
$$

$$
\operatorname{Hom}_{A}(M, N)=\left\{\phi \in \operatorname{Hom}_{B}(M, N):\left(\phi \otimes 1_{T}\right) P(M) \subseteq P(N)\right\} .
$$

(This is a slight modification of Theorem 4.2 of [6]).

Proof. Since $c$ is infinite, $c=2 c$ and, if $I$ is an index set with $|I|=c$, we can find free generators $s_{i}, t_{i}(i \in I)$ of $T$. Let $T^{\prime}$ be the subgroup generated by $\left\{t_{i}: i \in I\right\}$. Since $|\Gamma| \leqslant c$ we can find a subset $J \subseteq I$ with $|J|=|\Gamma|$ and use $J$ to index elements of $\Gamma$ so that $\Gamma=\left\{\gamma_{j}: j \in J\right\}$. We define also $\gamma_{i}=1$ for $i \in I \backslash J$.

For any $M \in{ }_{A} \mathfrak{M}$ we define $P(M)$ to be the additive subgroup of $M \otimes T$ generated by

$$
\left\{x \otimes s_{i}+\gamma_{i} x \otimes t_{i}: x \in M, i \in I\right\} .
$$

It is easy to verify that $P(M)$ is a $B$-submodule of $M \otimes T$ with the required properties.

LeMma 3 [6; Lemma 3.1]. Let $S$ be a free abelian group of rank 3. Then there exists a set $\mathscr{S}$ of four direct summands of $S$ such that for any abelian group $X$,

$$
\operatorname{Hom}((S ; \mathscr{S}),(X \otimes S ; X \otimes \mathscr{S}))=X \otimes 1_{s}
$$


(Here, and elsewhere, if $\mathscr{U}=\left\{U_{i}: 1 \leqslant i \leqslant n\right\}$ is a set of submodules of a module $U$ and $\mathscr{V}=\left\{V_{i}: 1 \leqslant i \leqslant n\right\}$ is a set of submodules of $V$, then

and

$$
X \otimes \mathscr{U}=\left\{X \otimes U_{i}: 1 \leqslant i \leqslant n\right\}
$$

$$
\left.\operatorname{Hom}((U ; \mathscr{U}),(V ; \mathscr{V}))=\left\{\phi \in \operatorname{Hom}(U, V): \phi U_{i} \subseteq V_{i}, 1 \leqslant i \leqslant n\right\} .\right)
$$

LEMMA 4 [6; Theorem 6.3]. Let $S$ be a free abelian group of rank 3 and $c<\aleph_{1}$ an infinite cardinal. Then there exists a free abelian group $F$ of rank $c$ and free direct summands $U$ and $U^{\prime}$ of $F \otimes S$ with $U \subseteq U^{\prime}$ and $U^{\prime} / U$ free of rank $c$, such that for every abelian group $X$,

$$
\left\{\phi \in \operatorname{Hom}(F, X \otimes F):\left(\phi \otimes 1_{s}\right) U \subseteq X \otimes U^{\prime}\right\}=X \otimes 1_{F} .
$$

We now proceed to the proof of Proposition 2. Choose $S, F, U, U^{\prime}$ as in Lemma 4. Let $T$ be a direct complement of $U$ in $U^{\prime}$ and use this group to define the functor $P$ as in Lemma 2. Define also

$$
W(M)=(M \otimes U) \oplus P(M) \subseteq M \otimes U^{\prime} .
$$

Then, exactly as in the proof of Theorem 6.4 of [6], we obtain, for $M, N \in{ }_{A} \mathfrak{M}$,

$\operatorname{Hom}_{A}(M, N) \otimes 1_{F}=\left\{\phi \in \operatorname{Hom}_{B}(M \otimes F, N \otimes F):\left(\phi \otimes 1_{s}\right) W(M) \subseteq W(N)\right\}$.

Now write $V=F \otimes S$ and let $\mathscr{S}$ be as in Lemma 3 so that, if $M \in_{A} \mathfrak{P}$,

$$
\mathscr{V}(M)=M \otimes F \otimes \mathscr{S} \cup\{W(M)\}
$$

is a set of five submodules of $M \otimes V$. It then follows, as in the proof of Theorem 2.4 of [6] that, if $M, N \in{ }_{A} \mathfrak{M}$, then

$$
\operatorname{Hom}_{B}(M \otimes V ; \mathscr{W}(M)),(N \otimes V ; \mathscr{W}(N))=\operatorname{Hom}_{A}(M, N) \otimes 1_{V}
$$

To complete the proof we define, for each $M \in_{A} \mathfrak{M}, L(M)$ to be the $\Lambda_{6}(B)$ module identified with the five submodule system $(M \otimes V ; \mathscr{W}(M))$.

\section{Construction of pathological modules}

Let $R$ be a principal ideal domain which is not a field nor a complete valuation ring, $A$ an $R$-algebra centrally generated over $R$ by $c<\aleph_{1}$ elements and such that $\mathfrak{N}=\left\{_{R} A\right\}$ is a Szekeres incomplete class. Then Theorem 1 asserts that there is an $R$-module $T(A)$ with $\operatorname{rank} T(A)=c \operatorname{rank}_{R} A$ such that

$$
\text { End }_{R} T(A)=\text { End }_{A} A \simeq A^{\text {op }}
$$

so that $T(A)$ has the same decomposition properties as ${ }_{A} A$.

The problem of constructing a pathological $R$-module is thus reduced to the problem of finding a suitable $R$-algebra $A$. Modules with pathologies (i) and (iii)-(vi) of $\S 1$ may be constructed in this manner.

For (i) we choose $A=R$ and regard it as an algebra with $c$ generators. Suitable algebras for the cases (iii)-(vi) have been constructed by Corner $[4,5]$, Corner and Crawley [7] and Osofsky [9]. (The algebras required for (iii)-(v) are collected together in [1], where it is shown that the constructions can be made over almost any ring without proper idempotents.) 
A similar method is used by Warfield [12] to construct other pathologies.

The proof of (ii) for the case of a commutative Noetherian ring $R$ which is not an Artinian principal ideal ring is given by Warfield [11]. For $R=k[t]$, a module with the required property is $\prod_{I} k[[t]]$, where $I$ is an arbitrary index set.

Another application of Theorem 1 to the construction of modules with the pathology (i) is the following:

Let $D$ be a division ring containing $k$ in its centre and centrally generated over $k$ by less than $\aleph_{1}$ elements. Then there exists an indecomposable $k[t]$-module with local endomorphism ring $E$ satisfying $E / \operatorname{rad} E=D$.

Proof. Let $A$ and $B$ be the localisations at $\langle t\rangle$ of $D[t]$ and $k[t]$, respectively. Then ${ }_{B} A$ is a free $B$-module and so $\{k[t]\}$ is Szekeres incomplete. Thus there is a $k[t]$-module with endomorphism ring $A$.

This result contrasts with the following well-known lemma which asserts that there are severe restrictions on the possible local endomorphism rings of $k[t]$-modules if we require also a nil radical.

Lemma 5. Let $R$ be a principal ideal ring and $X$ an $R$-module with $\operatorname{End}_{R} X$ local with nil radical. Then either $\operatorname{End}_{R} X$ is $R / p^{n} R$, for some prime $p$ of $R$ and some integer $n$, or it is the quotient field of $R$.

Proof. First suppose there is a prime $p \in R$ such that the corresponding multiplication $p$. belongs to the radical of $\operatorname{End}_{R} X$. Then $p$. is nilpotent and so $p^{m} X=0$ for some integer $m$. Thus $X$ is a torsion module of finite length and, since $X$ must be indecomposable, we have ${ }_{R} X=R / p^{n} R$ for some $n$.

If there is no prime $p$ for which $p$. belongs to the radical of $\operatorname{End}_{R} X$, then all multiplications are isomorphisms and $X$ is a vector space over the quotient field $Q$ of $R$. But then $\operatorname{dim}_{Q} X=1$, since otherwise $\operatorname{End}_{R} X=\operatorname{End}_{Q} X$ is not local.

\section{References}

1. S. Brenner, "Some modules with nearly prescribed endomorphism rings", J. Algebra, 23 (1972), 250-262.

2. - "Decomposition properties of some small diagrams of modules", Symposia Mathematica, 13 (1974), 128-142.

3. A. L. S. Corner, " Every countable reduced torsion-free ring is an endomorphism ring ", Proc. London Math. Soc., (3), 13 (1963), 687-710.

4. - "On a conjecture of Pierce concerning direct decomposition of Abelian Groups", Proc. Colloq. Abelian Groups (Tihany 1963) 43-48 (Akademiai Kiado, Budapest, 1964).

5. —_ "Additive Categories and a theorem of W. G. Leavitt", Bull. Amer. Math. Soc., 75 (1968), 78-82.

6. _ "Endomorphism algebras of large modules with distinguished submodules ", J. Algebra, 11 (1969), 155-185.

7. - and P. Crawley, "An abelian p-group without the isomorphic refinement property", Bull. Amer. Math. Soc., 74 (1968), 743-745.

8. A. Orsatti, "A class of rings which are endomorphism rings of some torsion free abelian groups ", Ann. Scuola Norm. Sup. Pisa (3), 23 (1969), 143-153.

9. B. L. Osofsky, "A remark on the Krull-Schmidt-Azumaya theorem ", Canad. Math. Bull., 13 (1970), 501-505.

10. G. Szekeres, “ Countable Abelian groups without torsion ”, Duke Math. J., 15 (1948), 293-306. 
11. R. B. Warfield, "Rings whose modules have nice decomposition properties ", Math. Z., 125 (1972), 187-192. 12. - "Countably generated modules over commutative Artinian rings ", Pacific J. Math.,

Department of Applied Mathematics and Theoretical Physics,

University of Liverpool,

England.

Mathematisches Institut der Universität Bonn, Federal Republic of Germany. 